\title{
Geant4 Based Space Radiation Application for Planar and Spherical Geometries
}

\author{
V. Satya Prakash \\ Department of Physics, Government Degree College, Medak, India
}

Email address:

satyaprakashvpet@yahoo.co.in

To cite this article:

V. Satya Prakash. Geant4 Based Space Radiation Application for Planar and Spherical Geometries. Advances in Applied Sciences. Vol. 2, No. 6, 2017, pp. 110-114. doi: 10.11648/j.aas.20170206.13

Received: November 4, 2017; Accepted: November 17, 2017; Published: December 18, 2017

\begin{abstract}
A space radiation application is developed based on Geant 4 tool kit. Even though there are many space radiation applications like MULASSIS (MUlti-LAyered Shielding SImulation Software); the application developed here offers more flexibility in choosing the physical models. It can be used to analyze the spherical geometries directly in addition to the planar geometries. In this paper both planar and spherical geometries of aluminum (Al), polyethylene (CH2) and liquid hydrogen (1H2) materials are analyzed with the help of dose-depth curves. The hydrogen, helium and Fe particle spectra of Galactic Cosmic Radiation (GCR) radiation are used in the estimation of doses. The total equivalent doses for the periods of $1 \mathrm{Yr}$ are evaluated for aluminum, polyethylene and liquid hydrogen materials respectively and the liquid hydrogen is found to be more effective shielding material. These doses are compared to NCRP 2000 (National Council on Radiation Protection and Measurements) career dose limits to analyze the radiation risk for astronauts. The doses in case of planar geometries are well below the career limits of male as well female astronauts of age $35 \mathrm{Yrs}$ but those that in case of spherical geometries are well above the career limits for female astronauts of the same age. Doses for spherical geometries are below the career limits of male astronauts of same age only when areal densities of shield materials reach $100 \mathrm{~g} / \mathrm{cm}^{2}$. The dose in spherical geometry is found to be $\sim 4.4$ times to that of planar geometry when Al material of $10 \mathrm{~g} / \mathrm{cm}^{2}$ is exposed to GCR hydrogen spectrum. This is in agreement with the estimations of previous studies. The effect of geometry on the dose levels received by astronauts is also analyzed. It is found that the reduction in dose is about $10 \%$ more in spherical geometry than in planar geometry of the $\mathrm{Al}$ material. This is observed when the Al material is exposed to GCR hydrogen spectrum and its thickness is varied from 10 $\mathrm{g} / \mathrm{cm}^{2}$ to $120 \mathrm{~g} / \mathrm{cm}^{2}$.
\end{abstract}

Keywords: Space Radiation, Geant4, Solar Energetic Particles, Galactic Cosmic Rays, Dose, Equivalent Dose

\section{Introduction}

Geant4 (Geometry and tracking) is a toolkit developed and maintained by Geant 4 international collaboration. It is developed for the Monte Carlo simulation of particle transport across the materials. It is a modular software written in $\mathrm{C}++$. It is capable of simulating interactions of various particles with various materials in wide energy range $[1,2,3,4]$. This makes the usage of Geant4 toolkit in diversified areas like medical physics, detector simulations and space radiation transport etc.

Space radiation is composed of three components: Galactic Cosmic Rays (GCR), Solar Energetic Particles (SPE) and Trapped Radiation. Galactic cosmic rays are composed of very energetic particles with energies up to $10^{20} \mathrm{eV}$. Galactic cosmic radiation includes $85 \%$ protons, $14 \%$ alpha particles and $1 \%$ heavy ions [5].

Sun usually emits protons and electrons of energies of order of few keV. But Sun occasionally emits protons and a small fraction of heavy ions in the form of Coronal Mass Ejections (CME). The energies of these particles are up to several $\mathrm{GeV}$. The emission of these particles continues from several hours to few days. Even though energies of these particles are lower than that of GCR particles, the intensity of these particles is very high.

The trapped radiation is the result of the interaction of both GCR particles and SPE particles with the earth's geomagnetic field. Trapped radiation appears around the 
earth in the form of van Allen belts. The inner belt consists of both protons and electrons whereas the outer belt consists of only electrons. The typical energies of the electrons are up to $7 \mathrm{MeV}$ whereas the proton energies are up to $600 \mathrm{MeV}$ [5]. Transport period through this radiation is very small compared to the overall journey period of deep space voyages.

Even though there are many space radiation applications, the application developed here has certain advantages. The Geant4 based application called MULASSIS is an engineering application which is ready to use but it is basically confined to the planar geometries $[6,7]$. There is also no freedom to choose variety of physical models. Since the planar geometry is only primitive in nature, it cannot be used for practical space radiation problems. Any practical space habitat structure should be a 3 dimensional one like cylinder or sphere etc. So while estimating the doses in the present application spherical geometries are also considered in addition to the planar geometries. The results of the planar and spherical geometries are also compared to study the influence of geometry on the dose levels received by the astronauts in the space radiation.

\section{Methods}

The appropriate physics lists are selected from Geant 4 tool kit for electromagnetic and hadronic interactions of space radiation separately. G4 Decay Physics is commonly used for both electromagnetic and hadronic interactions. For the electromagnetic interactions - G4 Standard EM Physicsoption3 is used whereas for the hadronic interactions - G4 Hadron Elastic Physics QGSP-BERT-HP, G4 Hadron Elastic Physics and G4 Ion QMD Physics are used. Bethe-Block model is used for the electromagnetic interactions of protons and alpha particles of energies up to $10 \mathrm{TeV}$. ICRU73 model is used for the electromagnetic interactions of heavy ions of energies up to $10 \mathrm{TeV}$. For hadronic interactions of protons Bertini Cascade Model is used up to the energies of $9.9 \mathrm{GeV}$, FTFP model is used up to the energies of $25 \mathrm{GeV}$ and QGSP model is used up to the energies of $100 \mathrm{TeV}$. For hadronic interactions of alpha particles and heavy ions - Binary Light Ion Cascade Model is used up to the energies of $110 \mathrm{MeV}$, QMD Model is used up to the energies of $10 \mathrm{GeV}$ and FTFP model is used up to the energies of $1 \mathrm{TeV}$.

Space radiation environments for GCR particles are chosen from SPENVIS (SPace ENVironment Information System) website [8]. From this basic data, General Particle Source (GPS) macros are prepared to make use in the present simulations. The number of particles coming out from the geometry under consideration is calculated from the differential particle flux spectra. This is done for the spectra of protons, alpha particles and Fe ions of CREME96 (Cosmic Ray Effects on Micro-Electronics) Solar Minimum GCR radiation [9, 10]. The Galactic cosmic Rays in CRÈME 96 model are given by the semi-empirical model of Nymmik et al. This model links the GCR intensity to activity in sun spots. This model incorporates solar activity cycle which reverses the polarity of solar magnetic field for every 11 Yrs.

The number of particles coming out from the surface area of $\mathrm{S}$ in the simulation setup is given by:

$$
N_{r}=\frac{1}{4} \Phi S
$$

where $\mathrm{S}$ is the area of the geometry under consideration in $m^{2}$ and $\Phi$ is the incoming integral particle flux in $m^{-2} s^{-1}$

The incoming integral particle flux $\Phi$ is evaluated using the differential particle flux histogram data taken from the SPENVIS website and integrating the linearized histogram function between the lower and upper limits of the energy spectrum.

The planar or spherical geometries are placed in a box filled with vacuum. The particles are generated either from a plane ahead of the shield slab or from a surface of the spherical shell enclosing the shell of shield material. Particle generation surfaces in both cases are considered to be of the same effective area even though the geometries are different. This makes the actual number of particles falling on each type of geometry the same. The distance between shield surface and particle generation surface is considered to be 25 $\mathrm{m}$ in both the cases. The particles are then generated randomly from these surfaces so that they incident isotropic ally on the shield geometries. A water slab or spherical shell of thickness $15 \mathrm{~cm}$ is placed immediately behind the shields of plane or spherical geometries respectively in order to compute the doses. This water phantom is considered to be equivalent to the human body in the present simulations. Doses are then converted to the equivalent doses following the recommendations of International Council for Radiological Protection (ICRP 60) [11]. Since the absorbed doses do not reveal the actual biological damage caused by different ionizing radiations, the equivalent doses are evaluated.

Dose or absorbed dose is calculated by using the following formula:

$$
\text { Dose }(D)=d E / d m(\text { in Gy) }
$$

where $\mathrm{dE}$ is the energy deposited by the radiation in a material and $\mathrm{dm}$ is the mass of the material exposed to the radiation

Equivalent Dose is defined as:

$$
\text { Equivalent Dose }(\mathrm{H})=w_{T} \text {.D (in Sv) }
$$

where $w_{T}$ is the radiation weighting factor

According to ICRP60 (International Commission on Radiological Protection), the $w_{T}$ value for protons is 5 and that for alpha particles and heavy ions is 20 . These values of $w_{T}$ are used in calculating the equivalent doses in the present simulations.

If $N_{s}$ is the number of particles simulated and $N_{r}$ is the real number of particles emerging from the given geometry due to the given GCR environment, then real dose $D_{r}$ is given by:

$$
D_{r}=\left(N_{r} / N_{s}\right) D_{s}
$$

where $D_{s}$ is the dose for $N_{s}$ particles used in the simulation. 
The real doses calculated from this formula are used in the equivalent dose calculations of the present study. The dose depth curves for individual ions from Figure 1-3 and Figure 5-7 are plotted only for $D_{s}$ values obtained in the simulations. The error bars are also included in these graphs.

According to the NCRP (2000) recommendations, the tenyear career dose limits in Lower Earth Orbit (LEO) for female and male astronauts of age $35 \mathrm{Yrs}$ are $60 \mathrm{~Sv}$ and 100 Sv respectively. Since there are no such recommended dose limits beyond LEO, the same limits are used for the comparison of simulation results and estimating the radiation risk for astronauts in deep space.

The doses in case of spherical geometry apply to all real space shuttle situations where space shuttle is exposed to isotropic space radiation environment. The slab geometry of shields is only applicable in situations where an instrument is placed immediately behind the space shuttle walls. The dose limits for sensitive electronic instruments are different from those suggested for astronauts, depending on the instrument and so on.

\section{Results}

\subsection{Results for Planar Geometry}

Sheets of $\mathrm{Al}$, polyethylene and liquid hydrogen with areal density varying from $10 \mathrm{~g} / \mathrm{cm}^{2}$ to $120 \mathrm{~g} / \mathrm{cm}^{2}$ are exposed to the GCR radiation spectra of hydrogen, helium and $\mathrm{Fe}$ ions. These ions are generated from the planes of same area as that of the shielding materials. Shield as well as source planes are of area $100 \times 78.50 \mathrm{~m}^{2}$. The distance between the source and shield planes is considered to be $25 \mathrm{~m}$. A Water slab of $15 \mathrm{~cm}$ thickness and area same as that of the shielding material is placed immediately behind the every shield slab and doses are then calculated for every 50000 ion species of GCR spectra. However, the doses for respective ions are normalized in the present work according to the actual number of those ions calculated from their differential energy spectra as well as the source geometry and angular considerations.

Doses in $\mathrm{Al}$ are high for all the three ions of GCR radiation. Doses in case of polyethylene are medium whereas those in case of liquid hydrogen are low. So polyethylene is more effective shielding material compared to $\mathrm{Al}$ and liquid hydrogen is more effective shielding material compared to polyethylene. This is due to the more density of target protons and small number of secondary particle production in liquid hydrogen material.

The annual equivalent doses estimated here are well below the NCRP (2000) career dose limits for all materials in case of male as well as female astronauts of age 35 Yrs.

The annual equivalent doses for $\mathrm{Al}$ and polyethylene at 10 $\mathrm{g} / \mathrm{cm}^{2}$ are in agreement within $10 \%$ with the Bernabeu and Casanova (2007). The difference in the results can be attributed to the difference in the simulation setup as well as the difference in choosing the physics models.

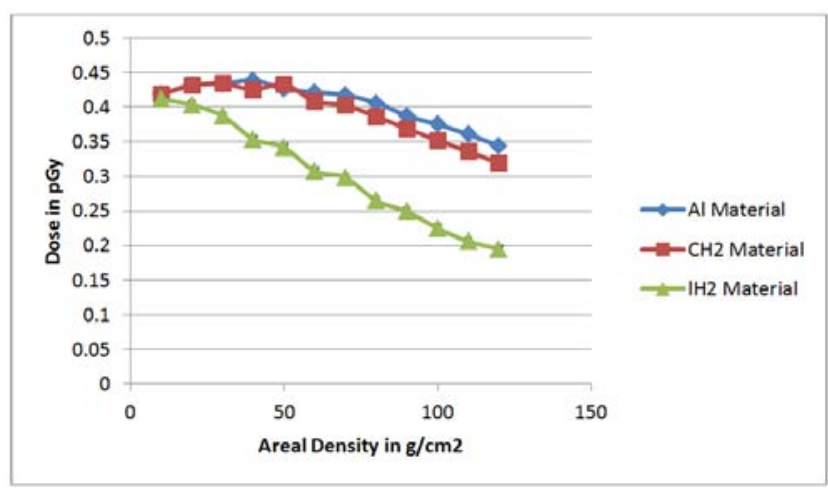

Figure 1. Comparison of doses in planar geometries of various materials for GCR H ions of CREME96.

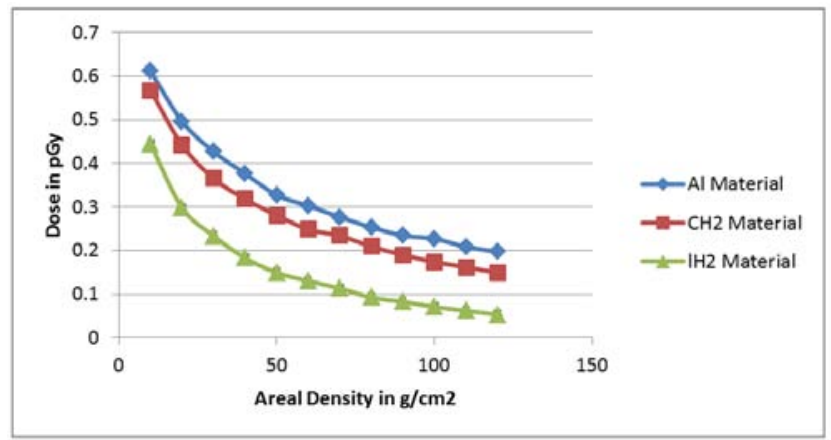

Figure 2. Comparison of doses in planar geometries of various materials for GCR He ions of CREME96.

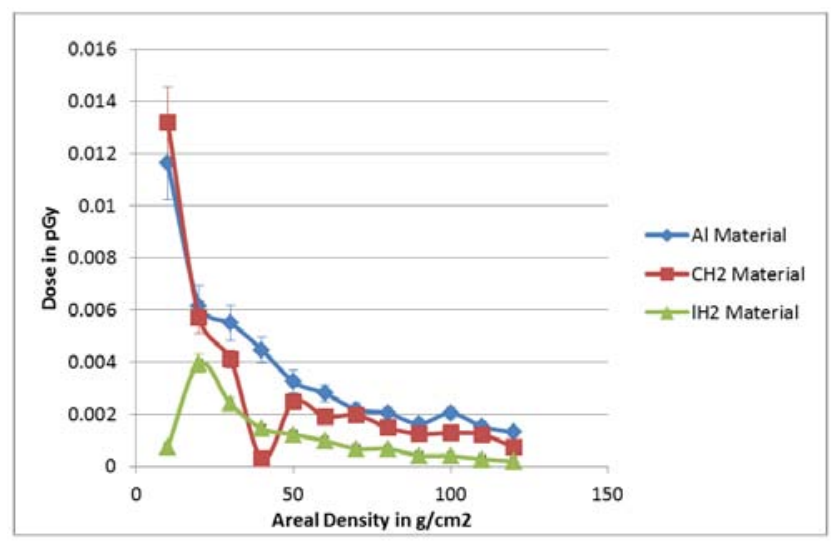

Figure 3. Comparison of doses in planar geometries of various materials for GCR Fe ions of CREME96.

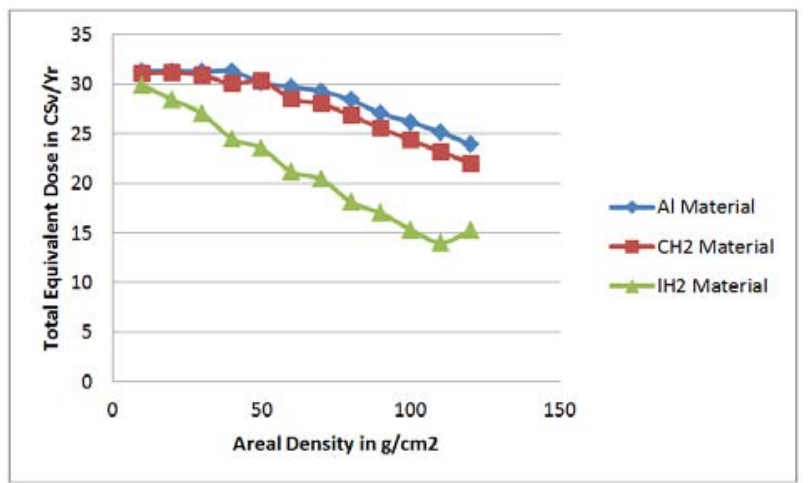

Figure 4. Comparison of total equivalent doses in planar geometries of various materials for GCR $\mathrm{H}$, He and Fe ions of CREME96. 


\subsection{Results for Spherical Geometry}

Spherical shells of $\mathrm{Al}$, polyethylene and liquid hydrogen with thickness varying from $10 \mathrm{~g} / \mathrm{cm}^{2}$ to $120 \mathrm{~g} / \mathrm{cm}^{2}$ are exposed to the GCR radiation environments of hydrogen, helium and $\mathrm{Fe}$ ions generated from the inner surfaces of spheres of radius $25 \mathrm{~m}$. The areas of corresponding spherical surfaces are of $7850 \mathrm{~m}^{2}$. The inner radii of the shield shells are taken to be $7.5 \mathrm{~m}$. Water shells of thickness $15 \mathrm{~cm}$ are placed immediately behind the shield shells and the doses are calculated for every 1000000 ion species of GCR radiation. However, the real doses for respective ions are normalized here according to the actual number of those ions calculated from their differential energy spectra, source geometry and angular considerations.

The absorbed doses for Al material appear to be high compared to other materials. The liquid hydrogen is, however, giving lower doses compared to other materials. So the total equivalent dose values show that polythene is more effective shielding material than aluminium and liquid hydrogen is more effective shielding material than polyethylene.

The annual equivalent doses in the graph indicate that dose values are exceeding the career limits of $35 \mathrm{Yr}$ female astronauts at all thicknesses of materials. However, the annual equivalent doses for all materials are below the recommended dose limits of male astronauts of the same age for the areal densities above $100 \mathrm{~g} / \mathrm{cm}^{2}$.

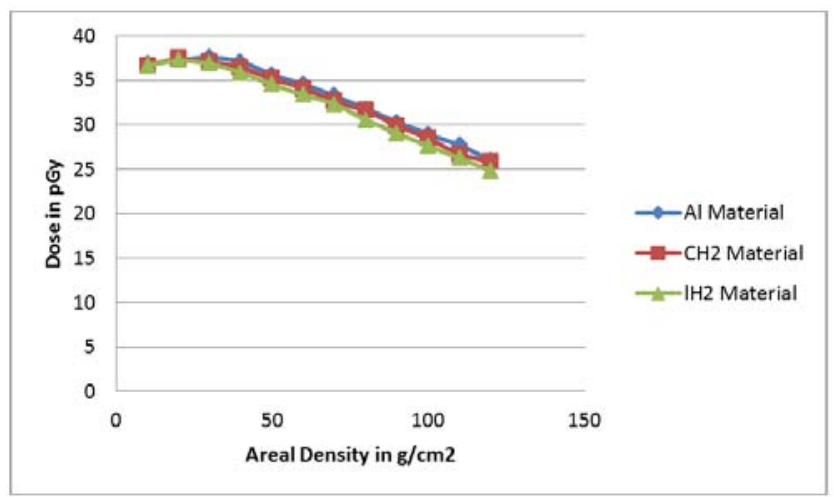

Figure 5. Comparison of doses in spherical geometries of various materials for GCR H ions of CREME96.

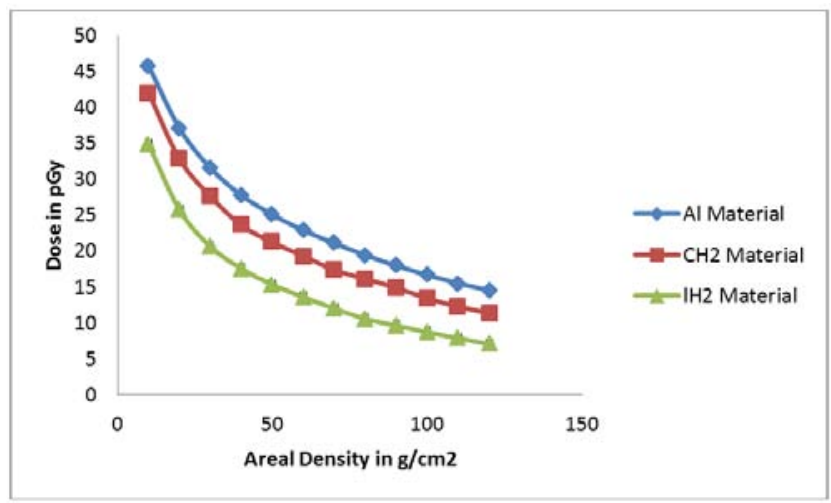

Figure 6. Comparison of doses in spherical geometries of various materials for GCR He ions of CREME96.

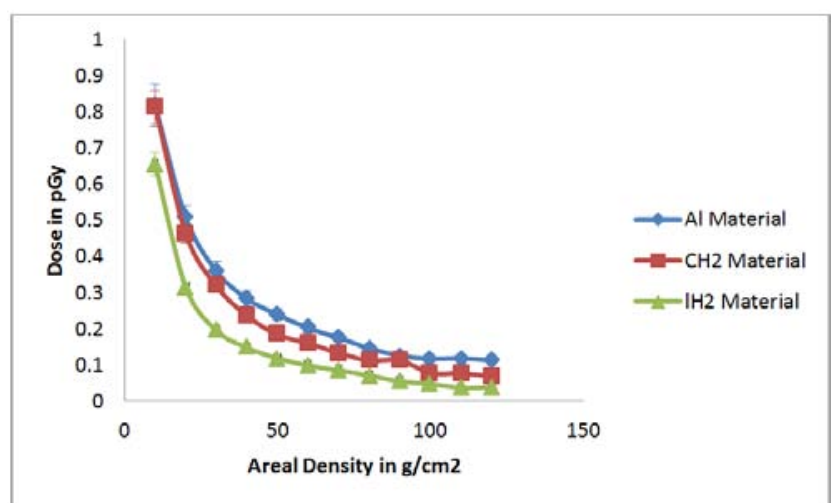

Figure 7. Comparison of doses in spherical geometries of various materials for GCR Fe ions of CREME96.

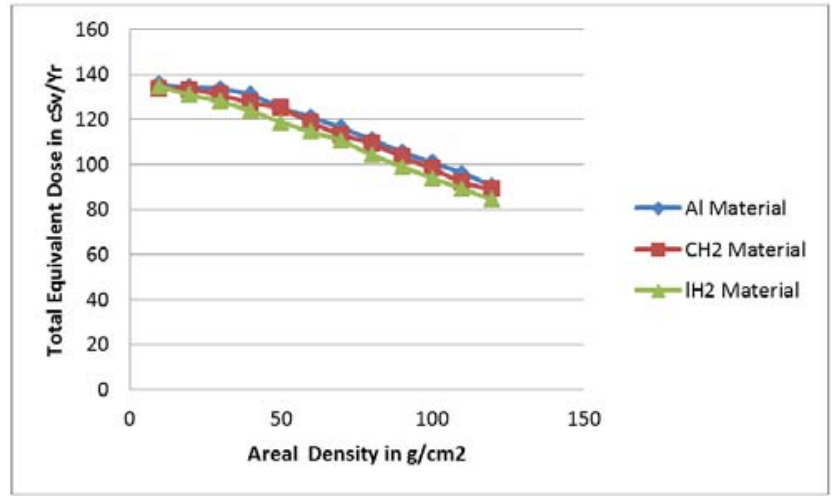

Figure 8. Comparison of total equivalent doses in spherical geometries of various materials for GCR H, He and Fe ions of CREME96.

\subsection{Comparison of Results of Planar and Spherical Geometries}

The doses in case of aluminium, polyethylene and liquid hydrogen are studied both in case of planar and spherical geometries. Doses in case of planar and spherical geometries of Al material of $10 \mathrm{~g} / \mathrm{cm}^{2}$ thickness are $5.5438 \mathrm{cGy} / \mathrm{Yr}$ and $24.3963 \mathrm{cGy} / \mathrm{Yr}$ respectively for GCR hydrogen ions. So the dose in case of spherical geometry is $\sim 4.4$ times the dose in case of planar geometry. This result is in agreement with the estimations in Seltzer [12]. The total equivalent doses in case of planar and spherical geometries of Al material of thickness $10 \mathrm{~g} / \mathrm{cm}^{2}$ are $31.28 \mathrm{cSv} / \mathrm{Yr}$ and $135.28 \mathrm{cSv} / \mathrm{Yr}$ respectively. So the total equivalent dose in case of spherical geometry is $\sim 4.3$ times the dose in case of slab geometry. The reason for the high doses in case of spherical geometries is that shields as well as phantoms are exposed to radiation from a spherical surface enclosing the shield surface. This leads to double penetration of incident particles through the phantoms.

The equivalent dose reduction in spherical geometry is about $33 \%$ in case of hydrogen ion exposure to $\mathrm{Al}$ over the thickness variation of $110 \mathrm{~g} / \mathrm{cm}^{2}$. The reduction of equivalent dose in planar geometry of the same material over the same thickness variation is about $23 \%$. The reason for this may be the effective path lengths traversed by the incident particles in the spherical geometries are more for a given areal density of the shield materials. 


\section{Conclusions}

The doses and total equivalent doses for $\mathrm{Al}$, polyethylene and liquid hydrogen materials are calculated from the GCR $\mathrm{H}, \mathrm{He}$ and $\mathrm{Fe}$ ion spectra of CREME96(Solar Min.). These values indicate that polyethylene is more effective shielding material than aluminium and liquid hydrogen is more effective shielding material than polyethylene. The reason for this is - secondary particle production in hydrogen rich materials is very low as expected. The doses in case of planar geometries are well below the career limits of male as well female astronauts of age 35Yrs whereas those in case of spherical geometries are well above the career limits for female astronauts of the same age. Doses in case of spherical geometries are below the career limits of male astronauts of same age only when areal densities of shield materials reach $100 \mathrm{~g} / \mathrm{cm}^{2}$. The dose in case of spherical geometry is $\sim 4.4$ times that in case of planar geometry for hydrogen ions in $\mathrm{Al}$ material of $10 \mathrm{~g} / \mathrm{cm}^{2}$ thickness. This is in agreement with the previous studies of Seltzer (1986).

The reduction of total equivalent dose in case of the spherical geometry is observed to be $\sim 10 \%$ more than that in case of planar geometry. The results in the present study are expected to be more realistic and exact, when compared to the previous MULASSIS based application of Bernabeu and Casanova (2007). This is due to the freedom to choose physics models as well as the facility to use spherical phantoms directly.

\section{Acknowledgements}

I wish to thank the Geant 4 collaboration for utilizing their Geant4 toolkit software version 4.10.1.p02 in the present simulations.

This work is supported by University Grants Commission (U.G.C) of India under Minor Research Project Grant No. F MRP- 4791/14 (SERO/UGC).

\section{References}

[1] S. Agostinelli, J. Allison, K. Amako et al., GEANT4- a simulation toolkit, Nucl. Instr and Meth. A, 506 (2003) 250303.

[2] J. Allison, K. Amako, J. Apostolakis et al., Geant4 developments and applications, IEEE Trans. Nucl. Sci., 53(1) (2006) 270-278.

[3] J. Apostolakis, M. Asai, A. G Bogdanov et al., Geometry and physics of the Geant 4 toolkit for high and medium energy applications, Radiation Physics and Chemistry, 78 (2009) 859873.

[4] Geant4. https://geant4.web.cern.ch/ [14 Oct 2015]

[5] M. Durante, and Francis A. Cucinotta, Physical basis of radiation protection in space travel, Rev. Mod. Phys, 83(4) (2011) 1245-1281.

[6] J. Bernabeu, I. Casanova, Geant4-based radiation hazard assessment for human exploration missions, Advances in Space Research, 40 (2007)1368-1380.

[7] F. Lei, P. R. Truscott, C. S. Dyer et al. MULASSIS: A Geant4based Multilayered Shielding Simulation Tool, IEEE Transactions on Nuclear Science, 49(6) (2002) 2788 - 2793.

[8] SPENVIS. https://www.spenvis.oma.be/

[9] Allan J. Tylka, James H. Adams, Jr., Paul R. Boberg, et al., CRÈME 96: A revision of the Cosmic Ray Effects on MicroElectronics Code, IEEE Transactions on Nuclear Science, 44(6) (1997) 2150-2160.

[10] CRÈME -MC. https://creme.isde.vanderbilt.edu

[11] ICRP1990 recommendations of the international commission on radiological protection, Tech. Rep. 60, International Commission on Radiological Protection (ICRP), New York, 1991.

[12] S. M. Seltzer, Conversion of depth-dose distributions from slab to spherical geometries for space-shielding applications, IEEE Transactions in Nuclear Science NS-33 (1986) 12921297. 